\title{
Editorial
}

\section{Mathematical Problems in Sustainable Energy and Environment}

\author{
Shengyong Chen, ${ }^{1}$ Maurizio Carlini, ${ }^{2}$ Nataliya Krasnogorskaya, ${ }^{3}$ and Massimo Scalia ${ }^{4}$ \\ ${ }^{1}$ College of Computer Science \& Technology, Zhejiang University of Technology, Hangzhou 310023, China \\ ${ }^{2}$ CIRDER, University of Tuscia, Via San Camillo de Lellis s.n.c., 01100 Viterbo, Italy \\ ${ }^{3}$ Department of Production Safety and Industrial Ecology, Ufa State Aviation Technical University, Karl Marx Street 12, \\ 450000 Ufa, Russia \\ ${ }^{4}$ Department of Mathematics, University of Rome "La Sapienza", Piazzale A. Moro 2, 00185 Rome, Italy \\ Correspondence should be addressed to Shengyong Chen; sy@ieee.org
}

Received 23 June 2013; Accepted 23 June 2013

Copyright (c) 2013 Shengyong Chen et al. This is an open access article distributed under the Creative Commons Attribution License, which permits unrestricted use, distribution, and reproduction in any medium, provided the original work is properly cited.

Achieving solutions to energy saving and environment protection problems that we face today requires longterm potential actions for sustainable development. Recent advances in this aspect have contributed to successful solutions to real problems, thus improving the sustainability of energy and environment and the quality of life. The focus of this special issue is on the mathematical models and solution methods to problems in sustainable energy, with modeling, analysis and control problems, communication systems, process control, environmental systems, intelligent manufacturing systems, transportation systems, structural systems, and so forth. A peculiar attention is paid to the related new development in rigorous mathematical physics definitions as well as intelligent methods for effectively solving the problems.

After a rigorous review process on 63 submissions, the special issue selects 23 papers which represent a good panel in the related new development, with the topics being connected with the mathematical problem models, elaborated solution methods, and their applications in real-world energy and environment systems.

In the category of energy production, D. Monarca et al. study the experimentation of innovative plants for electric power production using agroforest biomass and point out a connection among the chemical and physical properties of the outgoing syngas by biomass characterization and gas chromatography analysis. A. Petroselli et al. conduct tests on a PV-DC pump in Viterbo $\left(42^{\circ} 24^{\prime}\right.$ North, $12^{\circ} 06^{\prime}$ East) from January 2003 to November 2004, involve measurements of solar radiation, and develop a simple method that allows both the assessment of performances of the whole system and the optimal inclination angle of the panel. E. Bocci et al. simulate the power generation of a generating unit using the Rankine cycle and using refrigerant R245fa as a working fluid and illustrate the modeling of the system using TRNSYS platform, highlighting standard and "ad hoc" developed components as well as the global system efficiency. A. Marucci et al. analyze the radiometric properties of innovative semitransparent flexible photovoltaic materials in order to evaluate their performances in comparison with materials commonly used in the coverage of the greenhouses. M. Li et al. investigate the Hölder multiscales of sea level and reveal that the variations of sea level vary significantly at large time scale, and the dispersion of the Hölder exponents is usually spatial dependent. In another paper, M. Li et al. apply the correlation model to the autocovariance function (ACF) and the PSD of the sea surface wind speed observed in the Lake Worth, FL, USA, over the 1984-2006 period. $\mathrm{X}$. Zhang and Y. Zhang propose a method for calculating lightning transient responses on wind turbine towers. C. Yao et al. present a comparison of three wavelet neural networks for short-term forecasting of wind speed. C. H. Li proposes a novel modified Elman neural network for controlling permanent magnet synchronous generator systems. Z. Wang et al. analyze the dynamic responses of wind turbines under extreme working conditions, considering wind-rain loads. M. Xia et al. present a control strategy for microgrid containing renewable energy generations and electric vehicles.

In the category of energy utilization and saving, I. Garrido et al. investigate the performance of an ocean energy conversion system with DFIG sensorless control. H. Shi et al. adopt the divisible load theory for workload assignment in wireless 
sensor networks, which can prolong the energy depletion time of each sensor and make the whole wireless sensor network to operate for an extended duration. In order to increase the energy efficiency of radio access networks, S. Wen et al. propose a dynamic game model based on the controltheoretical approach to study the energy-efficiency issues in cooperative wireless cellular networks and use a predictor to supervise the data of imperfect channel state information. $\mathrm{N}$. Wu et al. present a decentralized data processing approach for structural health monitoring with smart wireless sensors by modal identification. J. Yan et al. build a nonlinear state space model for analyzing the highly nonlinear system and simplify the $\mathrm{H}-\mathrm{W}$ model into a linear-in-parameters structure. C. Yao et al. develops a novel diamond wire saw for formulation and implementation of energy-efficient ultraviolet curing. S. Cocchi et al. develop a model to simulate an air conditioning system with geothermal heat pump, which leads to optimization design of geothermal heat exchangers. S. Talatahari applies a bioinspired method, Ant Colony Optimization, to determine the optimum section of tunnels. Finally, Y.-J. Zheng et al. summarize the recent advances in bioinspired optimization methods, such as artificial neural networks and evolutionary algorithms, which are applied to the field of sustainable energy development.

In the category of environment protection and sustainability, M. Xia et al. propose a distributed beacon drifting detection algorithm to locate those accidentally moved beacons, which is a fundamental problem in wireless sensor networks, especially in unstable environments. To solve the cooperative game of global temperature lacking automaticity and emotional jamming, Z. Wang and J. Zhang develop an agent-based modelling method, which also uses a genetic algorithm to improve the investment strategy of each agent. E. Falatoonitoosi et al. examine the influential and important main green supply chain management practices, in particular green logistics and environmental protection.

Nevertheless, this special issue is not intended to constitute a complete compendium on the topic, but it does offer the reader an opportunity to appreciate the possibilities and the progress in the research area. It can be seen that although some models and solutions become available, most problems remain open and research is highly active in this field. We are aware of the present and upcoming technologies that will shape the future sustainable energy and environment, and we believe that all of us can contribute to the improvement of sustainability of our life styles.

\title{
Acknowledgments
}

As guest editors of this special issue, we would like to thank all the authors for their valuable contributions and the referees for their thorough reviews and helpful comments on the papers.

\author{
Shengyong Chen \\ Maurizio Carlini \\ Nataliya Krasnogorskaya \\ Massimo Scalia
}




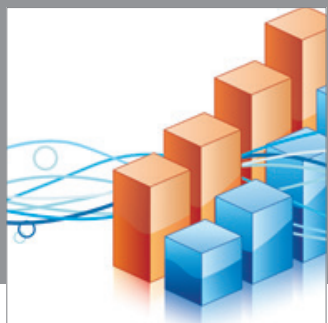

Advances in

Operations Research

mansans

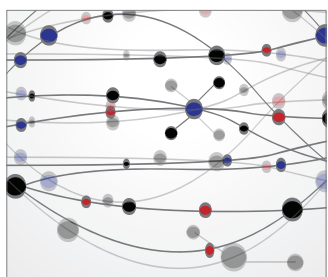

The Scientific World Journal
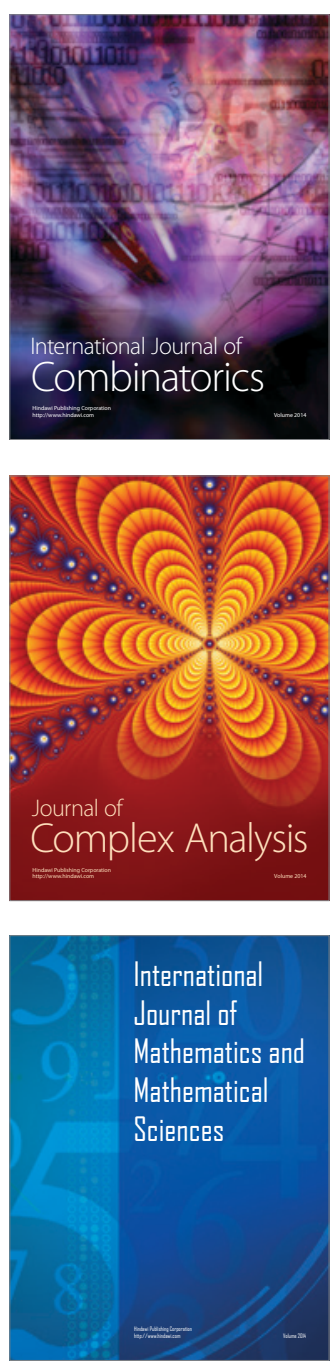
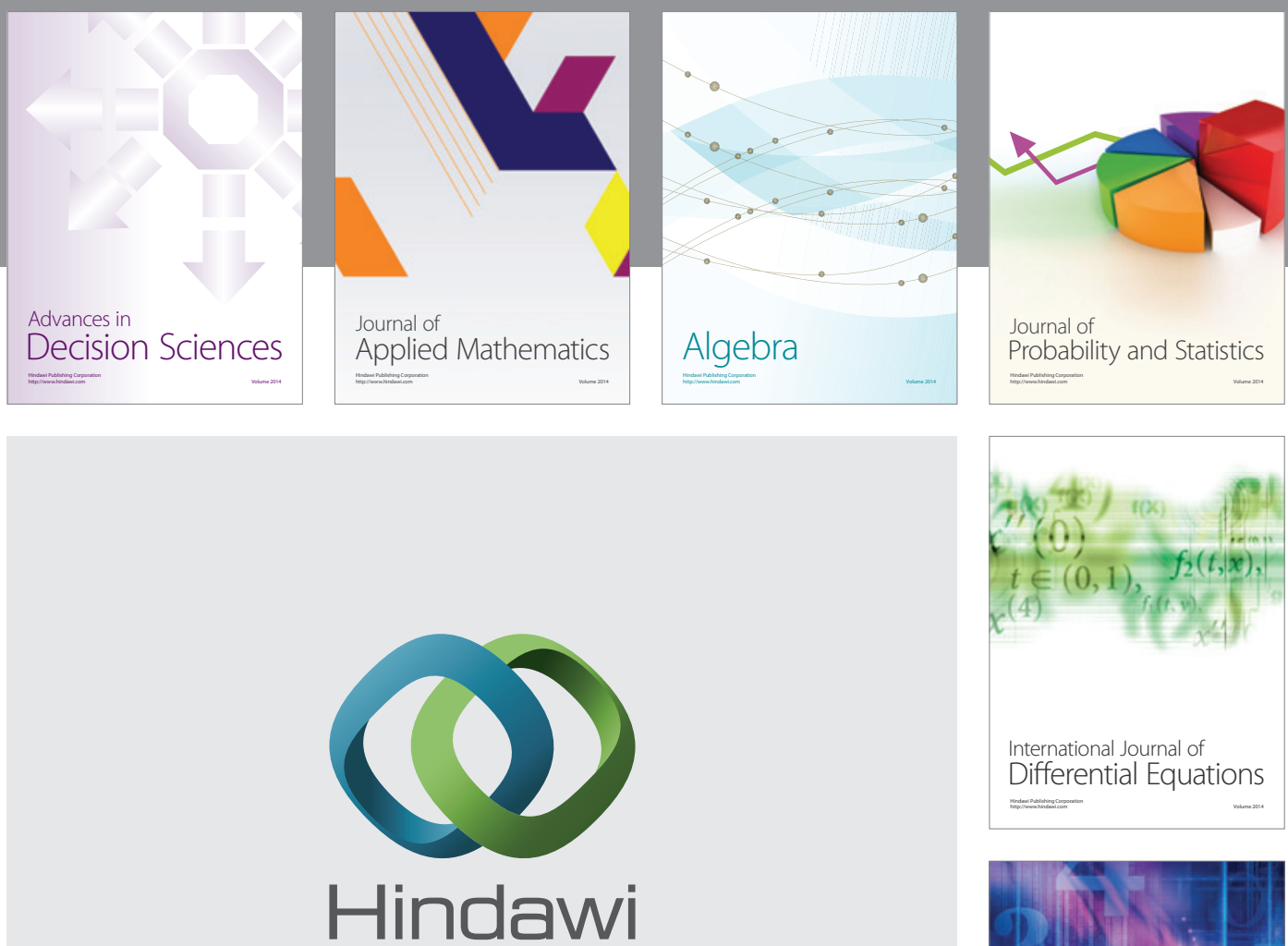

Submit your manuscripts at http://www.hindawi.com
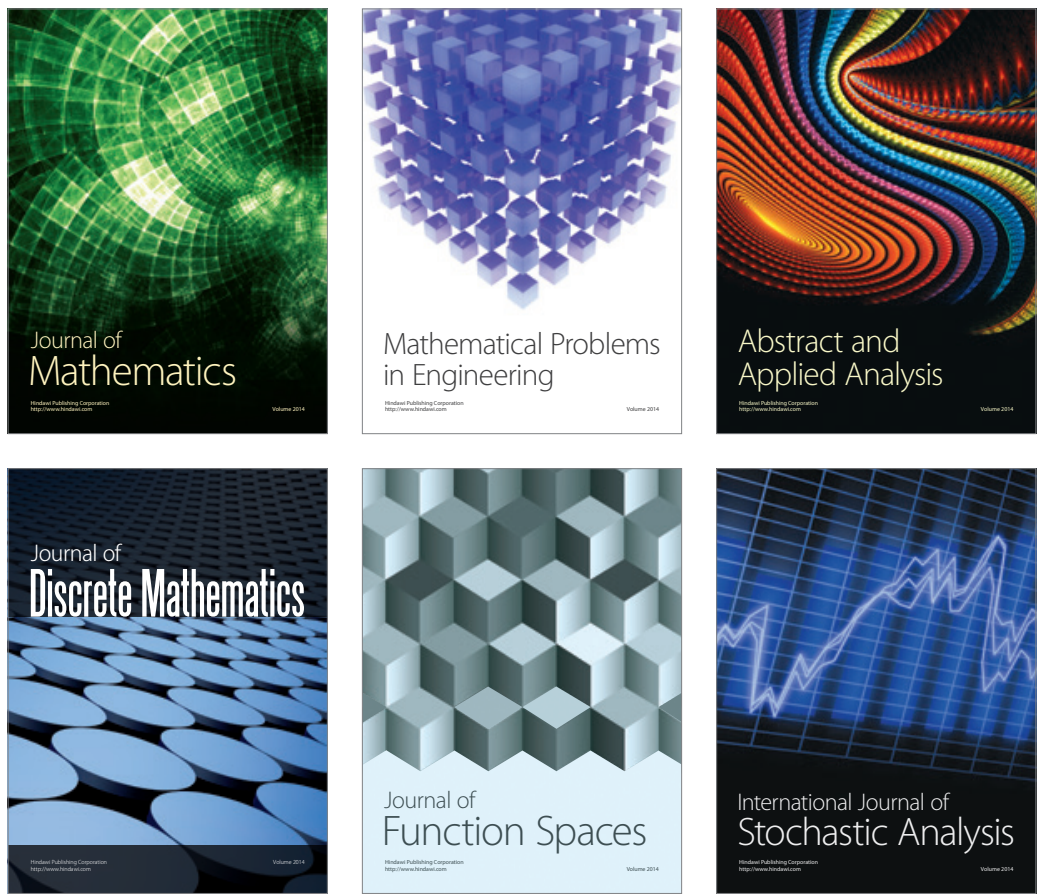

Journal of

Function Spaces

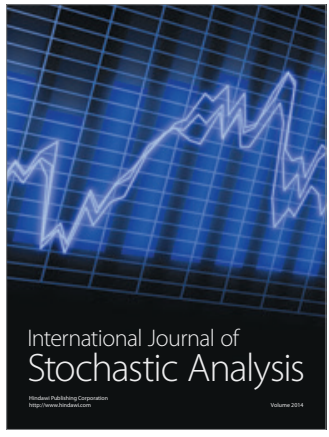

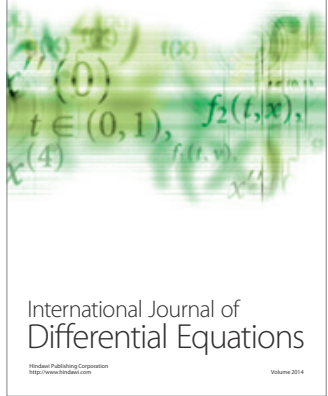
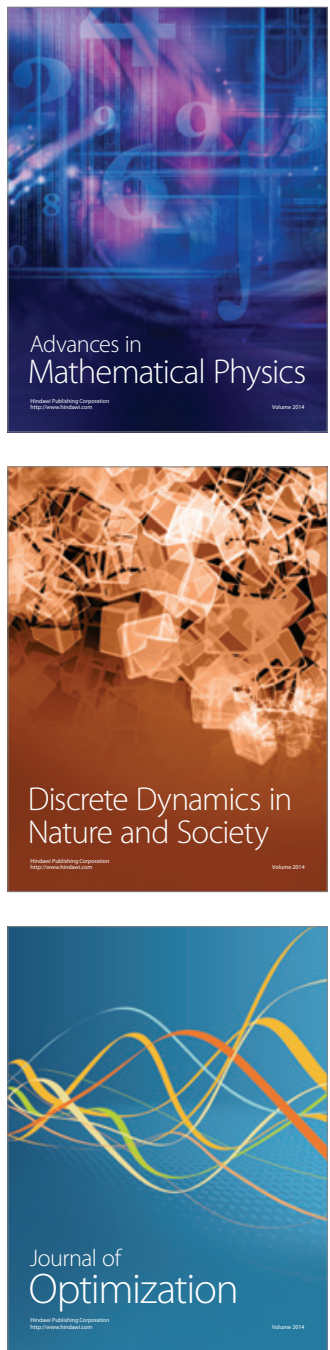